\title{
DESARROLLO INSTITUCIONAL EN LA IMPLEMENTACION DE ESTRATEGIAS DE CALIDAD EN EL PROGRAMA DE SEGURIDAD DEL PACIENTE EN COLOMBIA
}

\section{EDNA CAROLINA ORJUELA ESCOBAR}

Monografía para optar al título de Auditora en Salud.

UNIVERSIDAD SANTO TOMÁS

Especialización en Auditoría en Salud.

Bogotá, 2019. 


\title{
DESARROLLO INSTITUCIONAL EN LA IMPLEMENTACION DE ESTRATEGIAS DE CALIDAD EN EL PROGRAMA DE SEGURIDAD DEL PACIENTE EN COLOMBIA
}

\section{EDNA CAROLINA ORJUELA ESCOBAR}

Monografía para optar al título de Auditora en Salud.

\author{
Asesor \\ ALEJANDRA VALENZUELA CAZÉS \\ Fisioterapeuta \\ Esp. Docencia Universitaria \\ Mg en Salud Pública \\ UNIVERSIDAD SANTO TOMÁS \\ Especialización en Auditoría en Salud. \\ Bogotá, 2019.
}


Tabla de contenido

1. PLANTEAMIENTO DEL PROBLEMA …........................................................ 4

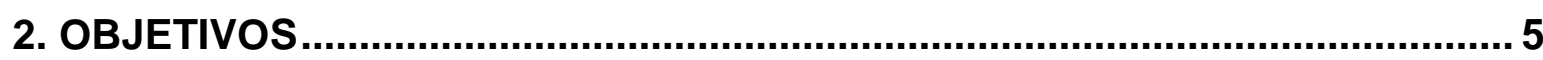

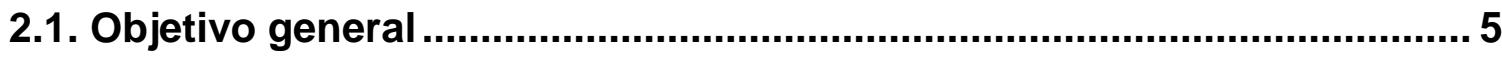

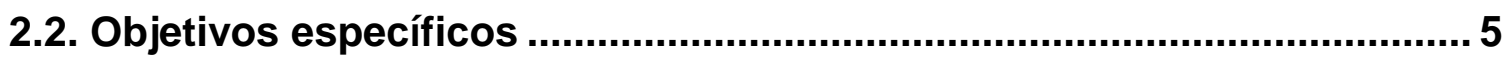

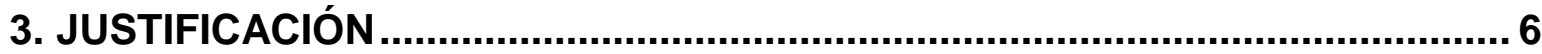

4. ELEMENTOS CONCEPTUALES …......................................................... 7

4.1. CONCEPTO DE SEGURIDAD DEL PACIENTE EN COLOMBIA ................ 7

4.2. Normatividad de Seguridad del Paciente en Colombia ${ }_{j} E_{\text {rror! }}$ Marcador no definido.

4.3. Estrategias de Calidad enfocadas a la calidad del paciente ................... 9

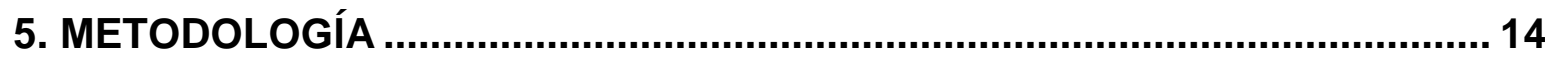

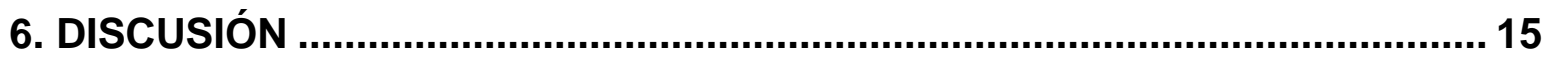

7. CONCLUSIONES Y RECOMENDACIONES ……............................................ 18

BIBLIOGRAFÍA 


\section{PLANTEAMIENTO DEL PROBLEMA}

Cualquier evento clínico que involucre a un paciente implica la posibilidad de desencadenar errores que pueden causar la culminación de la vida de este. Estos eventos adversos son un indicador significativo del resultado final de la atención y muestran, como ningún otro, cuál es la calidad de atención en una institución de salud.

Es necesario entender que el aseguramiento de la calidad de la atención del paciente en nuestro sistema es una problemática vigente a nivel mundial. Es por eso por lo que la OMS ha lanzado la "Alianza Mundial para la Seguridad del Paciente" para estandarizar las prácticas que brinden mucha más seguridad y eviten al máximo los errores en todas las entidades prestadoras de servicios de Salud.

Colombia trabaja de manera metódica en el mejoramiento de la seguridad del paciente, sin embargo, para poder notar resultados ha sido necesaria la implementación de varias acciones conjuntas que mostraron los beneficios que se otorgaron al paciente en este proceso. Entre estas acciones se encuentran la creación de un Sistema Obligatorio de Garantía de la Calidad y su impulso por parte del Ministerio de Salud Colombiano, mediante el Decreto 1011 de 2006.

Sin embargo, estas acciones, incluso coordinadas entre ellas, no son suficientes por sí solas para garantizar la calidad en la prestación de servicios de Salud, en nuestro país también es necesario un cambio cultural que permita ver las situaciones presentadas a diario desde el punto de vista del paciente y así, poder prestar el mejor servicio posible a cualquier persona que lo necesite, sea cual sea sus circunstancias.

Palabras claves: seguridad, paciente, calidad, estrategia. 


\section{OBJETIVOS}

\subsection{Objetivo general}

Análisis descriptivo de experiencias de la implementación del programa de seguridad del paciente en la actualidad

\subsection{Objetivos específicos}

- Revisión teórica del contexto teórico sobre seguridad del paciente

- Análisis de seguridad paciente como principio fundamental en la atención en salud

- Descripción a nivel institucional de la implementación de estrategias seguridad del paciente. 


\section{JUSTIFICACIÓN}

Colombia ha establecido e impulsado una Política de Seguridad del Paciente basado principalmente en el Sistema Obligatorio de Garantía de Calidad de la Atención en Salud cuyo principal pilar reside en la prevención de situaciones que afecten la seguridad del paciente, eliminando elementos adversos para constituir instituciones seguras y competitivas nacional e internacionalmente.

Para el crecimiento de este programa y el fortalecimiento de la seguridad del paciente en todas entidades prestadoras de servicios de salud es necesario entender que el desarrollo de una cultura de seguridad del paciente en todo el personal es de vital importancia, ya que este define la calidad de los servicios prestados y por esto se ha convertido en una competencia obligatoria en todos los involucrados.

La creación de esta cultura ha sido uno de los retos más grandes durante este crecimiento y por esto es necesario que todo el personal de la salud se adueñe de esta cultura, haciendo de esta su principal herramienta para prestar un buen servicio y con calidad. Sin embargo, esta cultura no solo debe ser exclusiva del personal médico sino de todo los involucrados en el proceso de prestación de servicios de salud, incluyendo pacientes, familiares y entidades.

Por tanto, este trabajo se fundamenta en la investigación del desarrollo de estrategias para el aseguramiento de la calidad en el programa de seguridad del paciente desde el punto de vista vivencial, haciendo un análisis de las estrategias implementadas para asegurar la calidad del programa de seguridad del paciente. 


\section{ELEMENTOS CONCEPTUALES}

\subsection{CONCEPTO DE SEGURIDAD DEL PACIENTE EN COLOMBIA}

En relación con el marco conceptual sobre la seguridad del paciente, existe un referente internacional conocido como la Organización Mundial de la Salud (OMS), quien en sus informes declara: la Seguridad del Paciente es un principio fundamental de la atención sanitaria. [1] Con respecto a lo anterior, se considera que dicho principio es obvio que debe desarrollarse en la atención de los usuarios, que tiene como fin lograr una atención libre de riesgos ante cualquier suceso adverso que esté propenso el paciente.

A nivel mundial, el reporte "To Erris Human: Building a safer Health System" del Instituto de Medicina de los Estados Unidos es reconocido como el documento que marcó el inicio al movimiento por la seguridad del paciente. Como consecuencia, la Organización Mundial de la Salud (OMS) creó la Alianza Mundial por la Seguridad del Paciente en 2004, lo que ayudó a que los países empezaran a trabajar en estrategias para evitar eventos adversos prevenibles y desde entonces se han generado gigantescos esfuerzos que se realizan para mejorar la seguridad de la atención y la evidencia de estos mediante las publicaciones internacionales. [3]

En Colombia se han realizado cambios para acoger estas medidas establecidas por la OMS y para esto el Ministerio de Salud y Protección Social define el concepto de Seguridad del Paciente como "el conjunto de elementos estructurales, procesos, instrumentos y metodologías basadas en evidencias científicamente probadas que propenden por minimizar el riesgo de sufrir un evento adverso en el proceso de atención de salud o de mitigar sus consecuencias. Implica la evaluación permanente de los riesgos asociados a la atención en salud para diseñar e implantar las barreras de seguridad necesarias. [4]

Hoy, una de las estrategias planteadas por el Ministerio de Salud para fomentar el cumplimiento de buenas prácticas en la prestación de servicios sanitarios es el requerimiento obligatorio para la acreditación de la implementación de programas de seguridad del paciente, así como de buenas prácticas para seguridad de este. [5]

Sin embargo, cualquier tipo de estrategia o metodología implementada para alcanzar estos objetivos, ya sea aquí o en cualquier parte del mundo, necesita de la colaboración de muchos factores para poder surgir. En este caso en particular, se hace más que necesario que se cree una cultura cuyo interés principal este centrado en la atención prestada al paciente y su seguridad. [6] 
Así, se puede considerar que la cultura de seguridad del paciente como un lineamiento fundamental que tiene un gran impacto en la calidad de la atención y que además se ha ido constituido gradualmente en un tema de interés general en toda el área de la salud. De aquí, la importancia de promover este concepto con el objetivo principal de brindar el máximo de satisfacción y bienestar de los pacientes.

[7]

En nuestro país, el término "cultura de seguridad del paciente" comprende desde el conocimiento teórico como la ejecución de planes e iniciativas que respondan a los lineamientos establecidos por la política de Seguridad del Paciente, la cual tiene origen en el Sistema Obligatorio de Garantía de Calidad de la Atención en Salud. [7]

\subsection{Principios:}

Este sistema tiene las siguientes características [8]:

- $\quad$ Accesibilidad: posibilidad que tiene cualquier usuario de utilizar los servicios de salud que le garantiza el SOGCS.

- $\quad$ Oportunidad: posibilidad que tiene un usuario de obtener los servicios que requiere sin que presenten retrasos que pongan en riesgo su vida o su salud.

- Seguridad: conjunto de elementos estructurales, procesos, instrumentos y metodologías, basadas en evidencias científicamente probadas, que propenden por minimizar el riesgo de sufrir un evento adverso en el proceso de atención de salud o de mitigar sus consecuencias.

- Pertinencia: grado en el cual los usuarios obtienen los servicios que requieren, de acuerdo con la evidencia científica, y sus efectos secundarios son menores que los beneficios potenciales.

- Continuidad: grado en el cual los usuarios reciben las intervenciones requeridas, mediante una secuencia lógica y racional de actividades, basada en el conocimiento científico.

Continuando con el propósito de mejorar la seguridad del paciente en nuestras instituciones, en la actualidad se ha optado por establecer como obligatorio tener una estructura exclusivamente dedicada a la seguridad del Paciente conformada por una Política de Seguridad, un Programa de Seguridad del Paciente y de definir procesos institucionales asistenciales seguros, tal como lo dicta la resolución 2003 de 2014. [9]

En la actualidad esta resolución define los procedimientos y condiciones de inscripción de los restadores de servicio de salud y de habilitación de servicios de salud, además adopta el manual de inscripción de prestadores y habilitación de servicios de salud. Establece los referentes mínimos para el funcionamiento de las 
entidades prestadoras de salud en el país que garanticen la seguridad de los pacientes en dichas instituciones. [10]

\subsection{Estrategias de Calidad enfocadas a la calidad del paciente}

El concepto de Calidad ha cambiado con el pasar del tiempo y se encuentra en una continua evolución. Según la International Standards Organization (ISO), la "Calidad es el grado en que las características de un producto o servicio cumplen los objetivos para los que fue creado." Para la Organización Mundial de la Salud, una atención en salud de Calidad es "asegurar que cada paciente reciba el conjunto de servicios diagnósticos y terapéuticos más adecuado para conseguir una atención sanitaria óptima, teniendo en cuenta todos los factores y los conocimientos del paciente y del servicio médico, y lograr el mejor resultado con el mínimo riesgo de efectos iatrogénicos y la máxima satisfacción del paciente con el proceso." [11] Sin embargo, es necesario notar que con el transcurrir del tiempo, la seguridad de la paciente enmarcada dentro de la noción de calidad también ha evolucionado conforme avanza el desarrollo científico y tecnológico. Además, para evitar la generación de errores fatales se ha propuesto crear una cultura de seguridad para el paciente que sirva para que las instituciones prestadoras de servicios de salud identifiquen las causas de estos eventos adversos, reconocer las fallas y aprender de las mismas, implementando estrategias de prevención y prácticas adecuadas para proporcionar servicios con calidad. [12]

En Colombia, el Gobierno Nacional ha diseñado unas estrategias básicas para promover la seguridad del paciente, asegurando la calidad de los servicios prestados y minimizando el número de eventos adversos que pueden darse en cualquier procedimiento médico. Estas se pueden definir como el conjunto de acciones sencillas impulsadas por el Ministerio de la Protección Social para ser implementadas por las instituciones prestadoras de servicios de salud para así enseñar a nuestros profesionales a ser más hábiles en identificar y prevenir los errores más frecuentes que suceden durante el proceso de atención, fomentando así progresivamente la cultura de seguridad del Paciente.

A través del tiempo, con el avance de los conceptos, en Colombia se definieron las siguientes estrategias: [12]

- Estrategia 1: Educativa.

Esta estrategia tiene como objetivo principal la formación de canales de información de la política de seguridad del paciente mediante la creación de entes informativos de la misma, además, la promoción de la formación e investigación sobre la cultura de la seguridad del paciente en los centros educativos y universidades. Por otro lado, inculca las acciones educativas dirigidas al paciente, sus familiares y la comunidad sobre este tema. 
- $\quad$ Estrategia 2: Promoción de herramientas organizacionales.

Esta estrategia se basa en la promoción de estudios de prevalencia en prestadores y aseguradores, creación y divulgación de herramientas prácticas entre esos los reportes intrainstitucionales, extrainstitucionales y rondas de seguridad.

- $\quad$ Estrategia 3: Coordinación de actores.

Esta estrategia consiguió la constitución del comité técnico para la seguridad del paciente y demás organismos de vigilancia y control de la política de seguridad del paciente. Además, se desarrollaron las normas técnicas a través de la Unidad Sectorial de Normalización en Salud y se generó el plan de incentivos dentro del marco del SOGCS.

- Estrategia 4: Estrategia de información.

Esta estrategia compone todas aquellas acciones que ayudan a la promoción de experiencias exitosas a nivel nacional e internacional, junto a los beneficios de esta política.

- Estrategia 5: Articulación de los componentes del SOGC.

Esta estrategia plantea a la seguridad del paciente como parte integral del SOGC y de sus componentes: la habilitación, insisto, como aquella herramienta básica estructural de cumplimiento; la auditoría como aquel componente que evalúa los procesos de atención e identifica fallas la calidad para mejorarla; el tercero, la acreditación como el sistema de excelencia voluntario par hospitales y aseguradores que quieran sobresalir del mercado por resultados excelentes; y el cuarto el Sistema de Información para la Calidad donde, a través de indicadores de resultado en la atención, informamos tanto a prestadores, para generar competencia por calidad, como a aseguradores para que escojan mejor a su red de prestadores, buenos hospitales, y a los usuarios para que a su vez puedan escoger los mejores aseguradores, los mejores hospitales donde quieren ser atendidos.[13] 


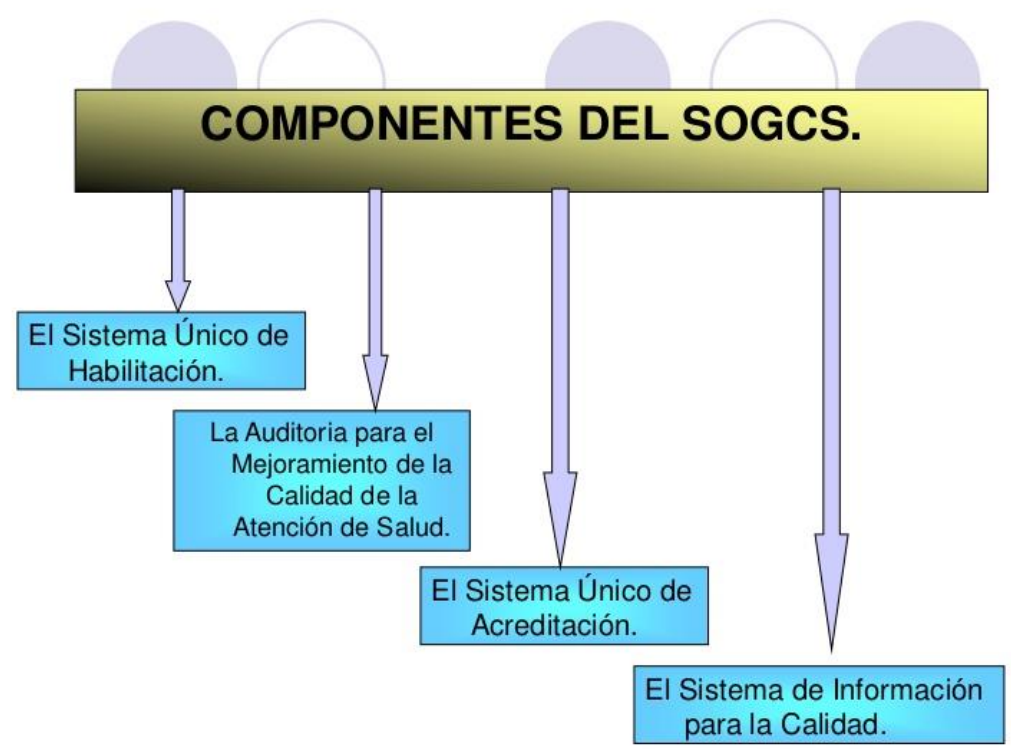

Imagen 1. Componentes del SOGCS. Ministerio de la Protección Social. República de Colombia. Decreto 1011 de 2006.

\subsection{Normatividad de Seguridad del Paciente en Colombia}

Actualmente, en Colombia existe una política Nacional de seguridad del paciente, encabezada por el Sistema Obligatorio de Garantía de Calidad de la Atención en Salud, el cual tiene como objetivo principal prevenir el acontecimiento de situaciones que afecten negativamente la seguridad del paciente, reducir, y de ser posible, eliminar la ocurrencia de eventos adversos para así contar con instituciones seguras y competitivas internacionalmente. [14]

Esta política ha sido fundamentada a través de los años en muchas normas, entre ellas encontramos:

La Constitución Política de la República de Colombia, promulgada en 1991, define la salud como un derecho fundamental de todos los colombianos. En el artículo 48 establece: "se garantiza a todos los habitantes del país el derecho irrenunciable a la seguridad social y a la salud, por lo cual el Estado deberá ampliar progresivamente la cobertura de seguridad social y deberá subsidiar a quienes, por escasez de recursos, no tiene acceso a los servicios de salud." [15]

Posteriormente, la ley 100 del año 1993, crea el Sistema de Seguridad Social Integral (SGSSS), la cual sería la reforma estructural para los servicios de salud de 
la época. En ella se define la Seguridad Social como: "el conjunto de instituciones, normas y procedimientos, que dispones las personas y la comunidad para gozar de una calidad de vida, mediante el cumplimiento progresivo de los planes y programas que el Estado y la sociedad desarrollen para proporcionar la cobertura integral de contingencias, especialmente las que menoscaban la salud y la capacidad económica de los habitantes del territorio nacional, con fin de lograr el bienestar individual y la integración de la comunidad."[16]

Hay que tener en cuenta que es la Ley 100 de 1993 el modelo de atención que rige en el ámbito de Seguridad Social en nuestro país, buscando los principios de eficiencia, universalidad, solidaridad, integralidad, unidad y participación. En el artículo 153 de esta ley, se establece la calidad como pilar principal del sistema: "El sistema establecerá mecanismos de control a los servicios para garantizar a los usuarios la calidad en la atención oportuna, personalizada, humanizada, integral, continua y de acuerdo con estándares aceptados en procedimientos y práctica profesional. De acuerdo con la reglamentación que expida el gobierno, las Instituciones Prestadoras deberán estar acreditadas ante las entidades de vigilancia." [16]

En el artículo 173 se instaura la formulación y adopción de forma coordinada de las políticas, estrategias, programas y proyectos para el sistema general de seguridad social en salud, cuyo principal objetico en enfocar la actitud gubernamental hacia la garantía de la seguridad del paciente. [16]

Se considera que la Ley 100 de 1993 es la base de la normatividad que rige nuestro sistema de seguridad social en salud, describiendo en su articulado los estándares de calidad y eficacia deseados en nuestro sistema de salud, siendo el punto de partida para el establecimiento de las estrategias, procesos y procedimientos en seguridad del paciente para la mejora de prestación de servicios por parte del equipo médico. [5]

En 1996, se publica la resolución 4445, donde se dictan las normas referentes al cumplimiento de las condiciones sanitarias y de seguridad a las que deben someterse las entidades hospitalarias y prestadoras de servicios de salud en el país, siendo de vital importancia su articulado en el contexto de seguridad del paciente, determinando estándares de manejo de infraestructura para la construcción de instalaciones, así como el diseño de suministros de agua, evacuación de todo tipo de residuo, disposición de unidades sanitarias, unidades de aseo, condiciones generales de pisos, cielo rasos, techos y paredes. Además, define características del área asistencial y de los servicios prestados en general por el equipo médico, haciendo énfasis en la importancia de éstas en el proceso de seguridad del paciente. [17]

El Decreto 2174 de 1996 organiza el sistema obligatorio de garantía de calidad en el Sistema General de Seguridad Social en Salud, fijando medidas que permitirían 
garantizar la calidad de la atención en salud en Colombia, basándose en los principios de accesibilidad, oportunidad, seguridad y racionalidad técnica. Se considera que es la primera norma que indica la característica de la seguridad en la atención en salud, garantizando los parámetros mínimos de calidad para el mejoramiento continuo de los servicios prestados por cualquier equipo médico en el país. [18]

En 1997, el Ministerio de Salud pública la resolución 0741 donde incita a las instituciones a establecer y desarrollar procesos administrativos necesarios de vigilancia y seguridad para la protección de los usuarios y trabajadores. Así, su principal objetivo es establecer las medidas mínimas que deben adoptar las instituciones prestadoras de servicios de salud para velar por la seguridad de los pacientes. [19]

El Decreto 2309 de 2002 definen "el conjunto de instituciones, normas, requisitos, mecanismos y procesos, deliberados y sistemáticos que desarrolla el sector salud para generar, mantener y mejorar la calidad de los servicios de salud en el país". Este está compuesto por el Sistema Único de Habilitación, la Auditoría para el mejoramiento de la Calidad de atención en salud, el Sistema Único de Acreditación y el Sistema de Información para la Calidad. [20]

En octubre de 2004 se establece la Alianza Mundial para la Seguridad del Paciente por parte de la Organización Mundial de la Salud con el propósito de "coordinar, difundir y acelerar las mejoras en materia de seguridad del paciente en todo el mundo". Esta se convierte en un motor que promueve la investigación en pro de una atención más segura buscando la reducción del riesgo de ocasionar daño a los pacientes de los estados miembros de la OMS, entre esos, Colombia. [21]

El Decreto de 1011 de 2006 establece el Sistema Obligatorio de Calidad de la Atención de Salud (SOGCS) del Sistema General de Seguridad Social en Salud, define los términos de importancia para la implantación de este, adiciona las acciones que desempeña el SOGCS, determina los componentes de este y las entidades responsables de su funcionamiento. [22] 


\section{METODOLOGÍA}

Recientemente y dentro del campo de la investigación ha surgido una nueva vertiente en la metodología de tipo cualitativo, denominada como "investigación narrativa". La investigación narrativa pretende establecer el narrar o contar historias como método de investigación y no como un proceso para llegar a este. [23]

Desde 1990 se empezó a sentir la necesidad de adquirir nuevas formas de generación y presentación de información y conocimiento viéndolo desde otra perspectiva; por lo que el termino narración comenzó a tener un rol visible en la investigación. [24]

Es así como la investigación narrativa tiene el eje de su análisis en la experiencia humana, más específicamente la investigación narrativa está dirigida al entendimiento y al hacer sentido de la experiencia [23]. Se considera entonces, como el estudio de la experiencia como un relato, entonces, es primero que nada y sobre todo una forma de pensar sobre la experiencia. La investigación narrativa como una metodología implica una visión del fenómeno. Usar la metodología de la investigación narrativa es adoptar una óptica narrativa particular que ve a la experiencia como el fenómeno bajo estudio [23]

Narrar significa poner en palabras lo que se ha vivido, resignificar las experiencias y darles sentido a acontecimientos. La descripción de experiencias nos permite conocer y comprender como transcurre lo vivido por otros en conjunto con sus interpretaciones, sentimientos, creencias, valoraciones e interacciones.

La autobiografía, los relatos de vida y la auto etnografía son características que la narrativa comparte con otros métodos de investigación dentro de la perspectiva cualitativa, así como el ámbito subjetivo y las experiencias personales. Los relatos nos permiten conocer de una persona o cultura y el escribir también es una forma de conocimiento, permitiendo que se elaboren textos reflexivos y experimentales que se alejan de la intención de producir leyes generales y universales y se acercan a lo concreto, lo específico, lo cotidiano y lo individual. [24]

Todas estas narraciones están basadas en fuentes de información. Las fuentes de información son aquellos tipos de documentos que contienen datos útiles para satisfacer una demanda de información o de conocimiento. [25]

Las fuentes primarias son aquellas que contienen información nueva u original. Se puede acceder a ellas directamente 0 a través de las fuentes secundarias. Entre estas se encuentran los libros y revistas convencionales.

Las fuentes secundarias son aquellas que contienen material ya conocido pero organizado según un esquema determinado. Estas nos remiten al material primario. 
Aplica técnicas de análisis documental para reorganizar la información y hacer accesible a los usuarios. Ejemplos: listas de resúmenes, índices.

Las fuentes terciarias son aquellas fuentes que contienen información de las fuentes secundarias y reproducciones de fuentes primarias. Ejemplos: bibliografía de bibliografías, fotocopias. [25]

\section{RESULTADOS}

Para la implementación del Programa Institucional de Seguridad del Paciente del se establecieron inicialmente 3 ejes orientadores:

- Estrategias para fomentar la cultura de seguridad del paciente

- Monitoreo de la Cultura de la seguridad del paciente

- Comité de Seguridad del Paciente

Dentro de las Estrategias para fomentar la cultura de seguridad del paciente se consideraron:

- Capacitación sobre Eventos Adversos, su prevención y detección oportuna a todo el personal con el fin de favorecer la "CULTURA DEL REPORTE".

- Educación continuada en: Prácticas seguras, prevención de infecciones nosocomiales.

- Hacer consciente al personal sobre la importancia de ofrecer al usuario un ambiente SEGURO. Estimulando y favoreciendo la RESPUESTA OPORTUNA ante un evento.

- Informando al usuario sobre sus Derechos y Deberes e involucrándolo en las soluciones, dentro de un ambiente de CONFIDENCIALIDAD Y CONFIANZA.

- Analizando uno a uno cada evento, asociándolos por causas e implementando estrategias de mejoramiento autogestionadas por los servicios.

- Elaborando planes de mejoramiento en cuanto: control de condiciones peligrosas en el ambiente físico, mitigación de Eventos Adversos, guías de manejo.

- Educación al usuario y su familia a través de folletos y cartillas en la cultura del auto cuidado.

Para el Monitoreo de la Cultura de Seguridad del Paciente, se realizará una encuesta de percepción anual a los trabajadores acerca de la seguridad del paciente 
en la Institución y así mismo se realizará análisis anual del incremento en el reporte de eventos.

El Comité de Seguridad del Paciente se encarga de liderar, dirigir, establecer lineamientos y analizar eventos reportados en materia de seguridad del paciente. Estará compuesto por el Gerente o su delegado, quien lo preside, Subgerente Científico, Auditor Médico, Asesor de Planeación y Subgerente Administrativo y Financiero y contará con un equipo operativo: Referente de Seguridad del Paciente, Coordinador Enfermería, Epidemiólogo, Infectó logo, Químico Farmacéutico, Referentes de los servicios, Auditor Médico, Gestor de Calidad, Ingeniero Biomédico, Referente Salud Ocupacional, Referente Gestión Ambiental y Coordinador de Mantenimiento.

Dicho comité realizará reuniones periódicas establecidas mensuales, en las cuales se invitará tanto personal asistencial como administrativo donde se realizará el debido análisis de los reportes de eventos adversos mediante un formato y utilizando la herramienta de análisis PROTOCOLO DE LONDRES, ESPINA DE PESCADO Y METODO SHELL. Allí se expondrá el caso, se revisará historia clínica, se analizará el evento y se tomaran las medidas correspondientes para dar solución y seguimiento al caso.

Se concretarán acciones y se dejara soporte en actas, que se darán a conocer a todos los integrantes previo a la siguiente reunión.

Específicamente el comité realiza las siguientes actividades:

- Diseñar nuevas estrategias de minimización del riesgo y analizar el grado de severidad, frecuencia y tipificación del evento en sí.

- Investigar e implementar las mejores prácticas existentes para el desarrollo de una cultura de seguridad de paciente al interior del hospital.

- Diseñar acciones específicas que fomenten y promueven la mitigación o disminución del nivel de ocurrencia de los incidentes reportados Esta actividad se hará en conjunto con el equipo operativo.

- Revisar, analizar, concluir el tipo de evento presentado y las acciones de mejoramiento a desarrollar, por parte de los responsables del proceso y/o procedimiento.

- Involucrar al personal de salud en el proceso de identificación y reporte voluntario, confidencial y de vigilancia activa.

- Fomentar la cultura del reporte de eventos adversos. 4. Presentar en comité la tendencia de la identificación de eventos debidamente clasificados por severidad, frecuencia, servicio, entre otros.

- Establecer el perfil de riesgo institucional debidamente tipificado, clasificado y servicio involucrado. Esta actividad se hará en conjunto con el equipo decisorio.

- Implementar el reporte intrainstitucional y extrainstitucional de eventos adversos, incidentes y eventos centinela, a través de la oficina de Planeación.

- Implementar Rondas de Seguridad en los diferentes servicios 
Habiéndose establecido lo anterior, se procederá a implementar las practicas seguras en la atención del paciente:

1. Identificación correcta del usuario:

Se colocará manilla de identificación a todo paciente que ingrese al servicio de urgencias desde su paso por admisiones y clasificados entre triage 2 y 3 , la cual deberá contener información correcta acerca de nombre completo del paciente, numero de historia clínica y fecha de nacimiento. El color de manilla para paciente estándar será color verde, para paciente psiquiátrico se colocará manilla roja, para $\mathrm{RN}$ se seguirá procedimiento establecido por la Institución. En caso de pérdida o deterioro, esta deberá ser cambiada, al alta del paciente deberá ser retirada por el personal ubicado a la salida de la Institución.

2. Prevención, detección y actuación ante riesgos de caídas:

Al ingreso del paciente a la institución con orden de hospitalización, paciente adulto mayor y/o menor de 14 de años y/o con alguna discapacidad, se diligenciará Formato Riesgo de Caída; el cual contendrá los datos generales del paciente que se deberán diligenciar completamente. Seguido a esto se encuentran especificados factores de riesgo que pueden generar un riesgo de caída, los cuales deben ser indagados en el paciente y marcados según corresponda. El formato se calificará según una escala numérica siendo 10/10 considerado como RIESGO ALTO y 1/10 RIESGO BAJO. Adicionalmente se agregará un consentimiento informado firmado por el paciente o acudiente confirmando conocimiento del riesgo y autorización para toma de medidas necesarias.

3. Identificación y registro de alergias a medicamentos:

Al ingreso del paciente a la institución se indagar y registrara debidamente cualquier alergia medicamentosa reportada, se diligenciará en la historia clínica con una alerta que nos indique cada vez que se ingrese a consultar la historia clínica, además se registrara en la manilla de identificación de manera clara y visible la alergia con un color llamativo.

4. Identificación del paciente e información relevante en la unidad

Al ingreso del paciente a la unidad se diligenciará el tablero del paciente ubicado sobre la cabecera y de manera visible al personal de salud, con Nombres y Apellidos completos, Numero de Identificación, Edad, Aseguradora, Riesgo de caída y Alergias reportadas (con diferente color). Para que todo el personal de salud pueda reconfirmar información. 


\section{DISCUSIÓN}

Desde un enfoque estatal, en cuanto la ejecución de la política de la seguridad del paciente en un país debe reflejarse de una manera sencilla y adecuada a las necesidades reales del sistema de salud. Que al implementarse dicha política se refleje de manera estable y sostenible con fines de asegurar unas buenas prácticas en una organización sanitaria.

De esta manera se pretende minimizar la incidencia de casos con una jerarquización de niveles de riesgos, el cual comprometa a los usuarios, además, promueve un excelente entorno de trabajo que motivador para el personal médico, lo que se concluye en gente orgullosa de hacer lo que hace de la manera que se hace.

Para la implementación del programa de seguridad del paciente debe tenerse en cuenta las siguientes variables: complejidad, extensión y vulnerabilidad del paciente.

Actualmente, la seguridad del paciente es una prioridad tanto en países desarrollados como subdesarrollados, entre esos el nuestro.

\section{CONCLUSIONES Y RECOMENDACIONES}

De la anterior investigación se puede concluir principalmente que nuestro país ha tenido un desarrollo exponencial en la creación e implementación de estrategias para asegurar la calidad de los servicios prestados por las instituciones de salud. Este éxito tiene como base la generación y aceptación de una cultura de seguridad del paciente mediante la creación de programas institucionales, los cuales se han convertido en un reto a través del tiempo.

Sin embargo, es necesario recalcar que este tipo de estrategias implementadas deben ser actualizadas al nivel de la ciencia y la tecnología para reducir los eventos adversos y preservar la vida de todos los pacientes otorgando servicios de calidad y es por esto por lo que la principal recomendación de este trabajo es buscar métodos de actualización de las estrategias establecidas o crear nuevas estrategias que con ayuda de los avances científicos se pueda reducir aún más la cantidad de eventos adversos en los procedimientos médicos actuales. 


\section{BIBLIOGRAFÍA}

1. Seguridad del Paciente [Internet]. Organización Mundial de la Salud. Actualizada 2019. Disponible en: http://www.who.int/topics/patient_safety/es/

2. Fajardo-Dolci, Germán Seguridad del paciente Cirugía y Cirujanos, vol. 78, núm. 5, septiembre-octubre, 2010, pp. 379-380 Academia Mexicana de Cirugía, A.C. Distrito Federal, México. [Internet]. Disponible en: https://www.redalyc.org/articulo.oa?id=66220238001

3. Seguridad del Paciente y atención segura. [Internet]. Ministerio de la Protección Social, versión 2.0. Disponible https://www.minsalud.gov.co/sites/rid/Lists/BibliotecaDigital/RIDE/DE/CA/Guiabuenas-practicas-seguridad-paciente.pdf

4. Buenas prácticas de seguridad del paciente. [Internet] Ministerio de Salud y Protección Social. Actualizado: Enero 16 de 2019. Disponible en: https://www.minsalud.gov.co/salud/CAS/Paginas/seguridad-del-paciente.aspx

5. "Estrategias para la implementación de la cultura de seguridad del paciente en el personal de enfermería." Lorena Rocío GonzalezAvila, Leidy Carolina Morales Soler, Robert Alexander Peñuela. Universidad Santo Tomás, Bogotá, 2018.

6.Promoción de la Cultura de Seguridad del Paciente. [Internet] Ministerio de Salud y Protección Social. Disponible https://www.minsalud.gov.co/sites/rid/Lists/BibliotecaDigital/RIDE/DE/CA/Guia_pro mocion_seguridad_paciente.pdf

7. "Una mirada actual de la cultura de seguridad del paciente". Olga Janneth Gómez Ramírez, Amparo Soto Gámez, Alejandra Arenas Gutiérrez, Jennifer Garzón Salamanca, Adriana González Vega, Erika Mateus Galeano. Av. enferm., Volumen 29, Número 2, p. 363-374, 2011. ISSN electrónico 2346-0261. ISSN impreso 01214500.

8.La Seguridad del Paciente, Un reto de Ciudad. [Internet] Alcaldía de Cali, Diciembre de 2012.2 Disponible en: http://calisaludable.cali.gov.co/seg_social/2013_Seguridad_Paciente/libro_segurid ad_del_paciente.pdf

9. Guía técnica de Buenas prácticas para la seguridad del paciente en la atención en salud, Ministerio de Salud, República de Colombia

10. Resolución 2003 de 2014.

11. Modelo de Calidad HIMFG. [Internet] Actualizado en 2016. Disponible en: http://himfg.com.mx/descargas/documentos/planeacion/plancalidad2016.pdf

12. Seguridad del paciente: Aspectos Generales y Conceptos Básicos. María Tatiana Espinal García, Luz María Yepes Gil, Luis Fernando García Gómez. Universidad CES, Medellín, Colombia. 2010.

13. "Herramientas para promover la estrategia de la seguridad del paciente en el Sistema Obligatorio de Garantía de Calidad de la Atención en Salud", Ministerio de la Protección Social, República de Colombia, 2007.

14. Seguridad del Paciente y atención segura. [Internet]. Ministerio de la Protección Social, versión 2.0. Disponible 
https://www.minsalud.gov.co/sites/rid/Lists/BibliotecaDigital/RIDE/DE/CA/Guiabuenas-practicas-seguridad-paciente.pdf 15. Constitución Política de la República de Colombia, 1991.

16. Ley 100 De 1993. Diciembre 23 De 1993. Diario Oficial No. 41.148.

17. Resolución Número 4445 De 1996

18. Decreto 2174 De 1996. Diciembre 3 de 1996. Diario Oficial No. 42.931

19. Resolución 0741 De 1997.

20. Decreto № 2309 de 2002.

21. Alianza Mundial para la Seguridad del Paciente- OMS Octubre 2004

22. Decreto 1011/2006 (3 Abril 2006) SOGCS

23. Investigación narrativa: una forma de generación de conocimientos. Mercedes Blanco. Argumentos (Méx.) vol.24 no.67 México sep./dic. 2011. [Internet] Disponible en: http://www.scielo.org.mx/scielo.php?script=sci_arttext\&pid=S018757952011000300007

24.Arias Cardona, Ana María; Alvarado Salgado, Sara Victoria. Investigación narrativa: apuesta metodológica para la construcción social de conocimientos científicos CES Psicología, vol. 8, núm. 2, julio-diciembre, 2015, pp. 171-181 Universidad CES Medellín, Colombia[Internet] Disponible en: https://www.redalyc.org/pdf/4235/423542417010.pdf

25. Fuentes de Información. [Internet] La Salle. Actualizado 2018. Disponible en: http://evirtual.lasalle.edu.co/info_basica/nuevos/guia/fuentesDelnformacion.pdf 